\title{
Patient-derived acute myeloid leukemia (AML) bone marrow cells display distinct intracellular kinase phosphorylation patterns
}

This article was published in the following Dove Press journal:

Cancer Management and Research

14 May 2009

Number of times this article has been viewed

\author{
Keith Shults' \\ Leanne Flye' \\ Lisa Green ${ }^{2}$ \\ Thomas Daly ${ }^{2}$ \\ Jason R Manro ${ }^{2}$ \\ Michael Lahn² \\ 'Esoterix, Brentwood, TN, USA; \\ 'Eli Lilly and Company, Lilly Research \\ Laboratories, Indianapolis, IN, USA
}

\begin{abstract}
Multiparametric analyses of phospho-protein activation in patients with acute myeloid leukemia (AML) offers a quantitative measure to monitor the activity of novel intracellular kinase (IK) inhibitors. As recent clinical investigation with FMS-like tyrosine-3 inhibitors demonstrated, targeting IK with selective inhibitors can have a modest clinical benefit. Because multiple IKs are active in patients with AML, multikinase inhibitors may provide the necessary inhibition profile to achieve a more sustained clinical benefit. We here describe a method of assessing the activation of several IKs by flow cytometry. In 40 different samples of patients with AML we observed hyper-activated phospho-proteins at baseline, which is modestly increased by adding stem cell factor to AML cells. Finally, AML cells had a significantly different phospho-protein profile compared with cells of the lymphocyte gate. In conclusion, our method offers a way to determine the activation status of multiple kinases in AML and hence is a reliable assay to evaluate the pharmacodynamic activity of novel multikinase inhibitors.
\end{abstract}

Keywords: leukemia, AML, bone marrow, flow cytometry

\section{Introduction}

Relapsed acute myeloid leukemia (AML) remains a disease with poor prognosis in which new therapies are needed. ${ }^{1}$ Targeting intracellular kinases (IK) responsible for critical signal transduction pathways in AML represents a novel approach of developing agents to treat AML. ${ }^{2}$ Clinically, activated or phosphorylated IKs are associated with prognosis in patients with AML. ${ }^{3}$ One important IK in AML is the FMS-like tyrosine kinase 3 (FLT 3). Various FLT3 inhibitors have been developed to inhibit wildtype or mutated FLT3. ${ }^{4-11}$ However, the clinical benefit of selective FLT3 inhibitors has been modest, perhaps because the single pathway inhibition may not be sufficient to affect the AML progression. Based on the observation that selective inhibition of FLT3 indirectly also modulates other IKs such as ERK, 8,12 novel IK inhibitors, such as KW $2449,{ }^{13}$ are being developed to target multiple IKs. For the clinical development of such multikinase inhibitors, it is essential to monitor sequentially the activity of IKs simultaneously.

One way to determine the status of IK activation and subsequent alteration by IK inhibitors, is based on the use of multiparametric flow cytometric analysis. ${ }^{14,15}$ This approach of analyzing multiple phosphorylated proteins in AML requires a sophisticated isolation, fixation and permeabilitization procedure followed by a rigorous flow cytometric analysis. Analyses protocols have been presented for the IK inhibitor sorafenib in AML patients, ${ }^{16}$ but such assessments are limited to specialized laboratories. ${ }^{17}$ 
Previous studies have suggested that assessing extracellular signaling-regulated kinase (pERK 1/2) may reflect antileukemia activity of sorafenib. ${ }^{16}$ Given the advances in flow cytometry, the role of IKs in AML pathology and the rapid development of several IK inhibitors, we here present a multiparametric flow cytometry assay, which can be conducted at routine clinical pathology laboratories. Our interest was focused on the assessment of pERK, pcKIT (stem cell factor receptor or CD117), pFLT3, pY100 (pan receptor tyrosine kinase marker), ribosomal protein pS6 (ribosomal protein involved in downstream signaling events of several receptor tyrosine kinases) and pAKT, because these IKs are generally activated in some or most AML cells., ${ }^{3,16}$ Thus, our assay may be used to monitor sequentially the activity of multi-targeted IK inhibitors in AML patients.

\section{Materials and methods}

\section{AML sample preparation (materials)}

In compliance with United States (US) Health Insurance Portability and Accountability Act (HIPAA) guidelines, we obtained bone marrow samples from 40 patients with relapsed AML from different hematology practices in the US. All samples were obtained as discarded material (during the course of 2006) along with patient specific phenotyping results, from the Esoterix Oncology diagnostic lab (Austin, TX) after all diagnostic testing had been performed and patient information had been de-identified. Demographic information noted on requisition forms was used to characterize the origin of the bone marrow samples. In each case, automated cell counts were obtained and samples were placed in a $37{ }^{\circ} \mathrm{C} / 5 \% \mathrm{CO}_{2}$ incubator for one hour prior to processing to allow enzyme activity and cellular processes to stabilize. The testing for each sample was obtained for basal levels and an additional aliquot was stimulated with $10 \mathrm{ng} / \mathrm{ml}$ of stem cell factor (R\&D Systems, Minneapolis, MN) for exactly 15 minutes and immediately fixed/permeabilized per US Patent \#7326577. All samples were then placed at 2-8 ${ }^{\circ} \mathrm{C}$ overnight to be stained for phosphorylated proteins the following day as indicated below.

\section{Antibodies (materials)}

All antibodies to phosphorylated proteins were obtained from Cell Signaling Technology (CST) (Danvers, MA). Antibody specificity titrations were performed prior to use of the antibodies based on rested and stimulated conditions to obtain the saturating concentrations for each antibody. Thus, the antibodies were used per $1-2 \times 10^{6}$ cells. The specific antibodies for this study were: phospho-FLT3 (CD135)
(Y591)-Alexa Fluor 488 (CST \#3459); phospho-c-KIT (CD117) (Y719) (CST\#3391L); phospho-p44/42 extracellular signaling-regulated kinase 1/2 (ERK1/2) (T202/Y204) Alexa Fluor 647 (CST\#4375); phospho-AKT (S473)-Alexa Fluor 647 (CST2337); phospho-Tyrosine-Y100-Alexa Fluor 488 (CST 9414); phospho-S6 Ribosomal Protein (S235/236)Alexa Fluor 647 (CST, Esoterix custom conjugate). The surface antibodies used for the study were CD11b-Alexa Fluor 700 (Esoterix custom conjugate; Beckman Coulter, Miami, FL); CD34-Biotin (Invitrogen, Carlsbad, CA) and CD45-PE (Immunotech; Beckman Coulter). The isotype controls used for the phospho-proteins were matched to the primary antibodies at the identical concentrations used in the assay. The CD34-Biotin was developed post fixation using streptavidin QDOT 605 (Invitrogen) and the phospho c-KIT was developed using a goat anti rabbit Alexa Fluor 488 (both used at $1 \mu \mathrm{g}$ per staining reaction). In all cases, the staining reactions were carried out in $50 \mu \mathrm{l}$ total volumes in phosphate-buffered saline (PBS) with $3.4 \%$ bovine serum albumin (BSA).

\section{Staining of AML samples for surface and phosphorylated proteins}

The samples were surface stained with the CD34 and CD11b antibodies for 20 minutes. If cells were chosen for ex vivo stimulation, they were immediately stimulated with SCF and fixed/permeabilized as indicated above. The cells were spun out of fixative and washed twice in PBS $+2 \%$ NCS. Respective phospho antibodies as listed below were added to the cell pellet and allowed to incubate for one hour at ambient temperature in the dark. The excess antibody was removed by washing the cells twice in $2 \mathrm{ml}$ of PBS-2\% NCS with 10 minute soaks between each centrifugation. The following combinations were used for each donor: Tube (1) p-cKIT/ p44/42/ERK1/2-AF647; Tube (2) pFLT3-AF488/pS6Ribosomal Protein-AF647; Tube (3) pY100-AF488/pAKTAF647; Tube (4) isotype Alexa 488/isotype Alexa 647. The secondary antibodies were added to the appropriate tubes in conjunction with CD45-PE in all tubes and incubated for an additional 20 minutes. The excess antibody was removed using same wash sequence as listed above. Finally, the cells were re-suspended in $400 \mu \mathrm{l}$ of PBS-2\% NCS final volume and 100,000 intact events were collected per tube during flow cytometry assessments.

\section{Cytometer information}

Flow cytometry was performed on a Beckman Coulter FC500 with custom filter sets designed to optimize the collection of 
Alexa 647 and Alexa 700 signals. The instrument was set up using QC3 beads (Bangs Laboratories, Fishers, IN) for the daily monitoring of instrument performance within an accepted "window of analysis". Full spectrum fluorescent calibration particles (Spherotech, Lake Forest, IL) were also run with each assay to determine the molecules of equivalent fluorescence (MEFL) of the phospho-proteins in a methodology that has been described in detail. ${ }^{18}$

\section{Sample analysis}

Each sample was analyzed using a direct data exchange (DDE) link between WinList 6.0 (Verity Software House, Topsham, MA) and Mircosoft Excel (Microsoft, Redmond, WA). All data gating, displaying, and processing were conducted in WinList. The pattern recognition of the marrow elements was by eye and experience based on known CD45/SSC patterns. The MEFL of the phospho-proteins was measured in the "blast" population as determined by CD45/SSC and/or CD34+, CD11b staining. In samples that contained more than one phenotypic clone of "blasts", each clone was analyzed individually for phospho-protein expression. The SCF stimulated levels were compared to the unstimulated levels in each population.

\section{Statistical analysis}

AML phospho-protein expression was analyzed using a mixed effect ANOVA model using the REML method to estimate covariance parameters. A variance components structure was used for the covariance matrix. For normalized untreated AML, the SCF-treated AML, and the ratio of untreated AML to lymphocyte responses the model used the following terms: AML FAB Classification, Patient nested within AML FAB Classification (random factor), IK, phenotype, IK* phenotype, AML FAB Classification*IK, and AML FAB Classification*phenotype. Pairwise comparisons were made using the LSMEANS and pdiff options in SAS Proc Mixed (SAS Institute Inc. Cary, NC). A Fisher's LSD approach was used to approximately control the experiment-wise type I error rate. Also, not all pairwise comparisons were of interest. For significant interaction model terms, the slice option was used in SAS to further protect the experimentwise error rate. Only statistically significant slices were followed up with further pairwise comparisons. The data were analyzed using JMP ver. 6.0.2 and SAS ver. 9.1 (SAS Institute, Inc.).

\section{Results}

We obtained bone marrow samples from 40 patients with relapsed AML to test a novel technique of determining phospho-protein expression in AML blasts and to profile AML blast cells based on their ability to express specific phospho-proteins. All samples were received from different hematology practices in the United States, and were evaluated as part of routine hematological evaluation (Table 1). We used samples from relapsed AML patients based on the assumption that AML blasts in advanced disease will show increased phosphorylation levels. ${ }^{16}$ From the 40 samples, $19(47 \%)$ had not been classified based on the FAB AML classification, of which eight patients had recurrent AML $(8 / 19 ; 42 \%)$. In addition to AML samples with no clear FAB classification, there were samples from six M1 and M4 AML patients, respectively (each 6/40; 15\%). For M2, M3, and M5 AML there were each three samples (3/40; 7\%). In $5 / 40(12 \%)$ patients the bone marrow showed less than $20 \%$ blast cells.

Based on this randomly collected material from patients with AML, we evaluated the phospho-protein levels without and with SCF ex vivo stimulation. After gating cells on forward scatter (FS) vs. side scatter (SS), cells were gated based on CD45, CD11b and CD34 expression prior to analyzing the phospho-protein expression of pERK, pc-KIT, pAKT, pY100, pFLT3, and pS6 (Figures 1 and 2). Based on the surface marker expression, we categorized the cell population in the following groups: (a) AML phenotype 1, (b) phenotype 2 and (c) lymphocytes. AML phenotype 1 was characterized by $\mathrm{CD} 34^{\text {hi }} / \mathrm{CD} 45^{\text {low }} / \mathrm{CD} 11 \mathrm{~b}$ - surface expression (red gate in Figures 2A,B) consistent with an "immaturelike" cell population. AML phenotype 2 was characterized by $\mathrm{CD} 34^{\text {low }} / \mathrm{CD} 45^{\mathrm{hi}} / \mathrm{CD} 11 \mathrm{~b}^{+}$surface expression (green gate in Figures 2A, B) which is characteristic for "mature-like" cells. Most samples in this study were AML Phenotype 1 cells (Table 2). Compared to the lymphocytes, both phenotype 1 and 2 AML cells showed high phospho-protein expression (Figures 2-4). Ex vivo stimulation with SCF increased levels of phosphorylation in both phenotypes of AML, but not in lymphocytes (Figures 2C-F). Hence, lymphocytes can serve as negative control for assessing the effect of SCF-induced phosphorylation of IKs in AML cells.

We then investigated whether phenotype $1 / 2$ or cells based on the FAB AML classification were associated with a particular phospho-protein expression pattern (Figures 3, 4). AML cells were immediately assessed after receipt in the clinical pathology lab and without any additional in vitro stimulation (basal condition) (Figure 3). pFLT3, pS6, pY100 and pcKIT were increased compared with $\mathrm{p}$-AKT and $\mathrm{p}$-ERK (Figure 3, Panel A and B). Phospho-protein expression between phenotype 1 and 2 was similar with statistical 


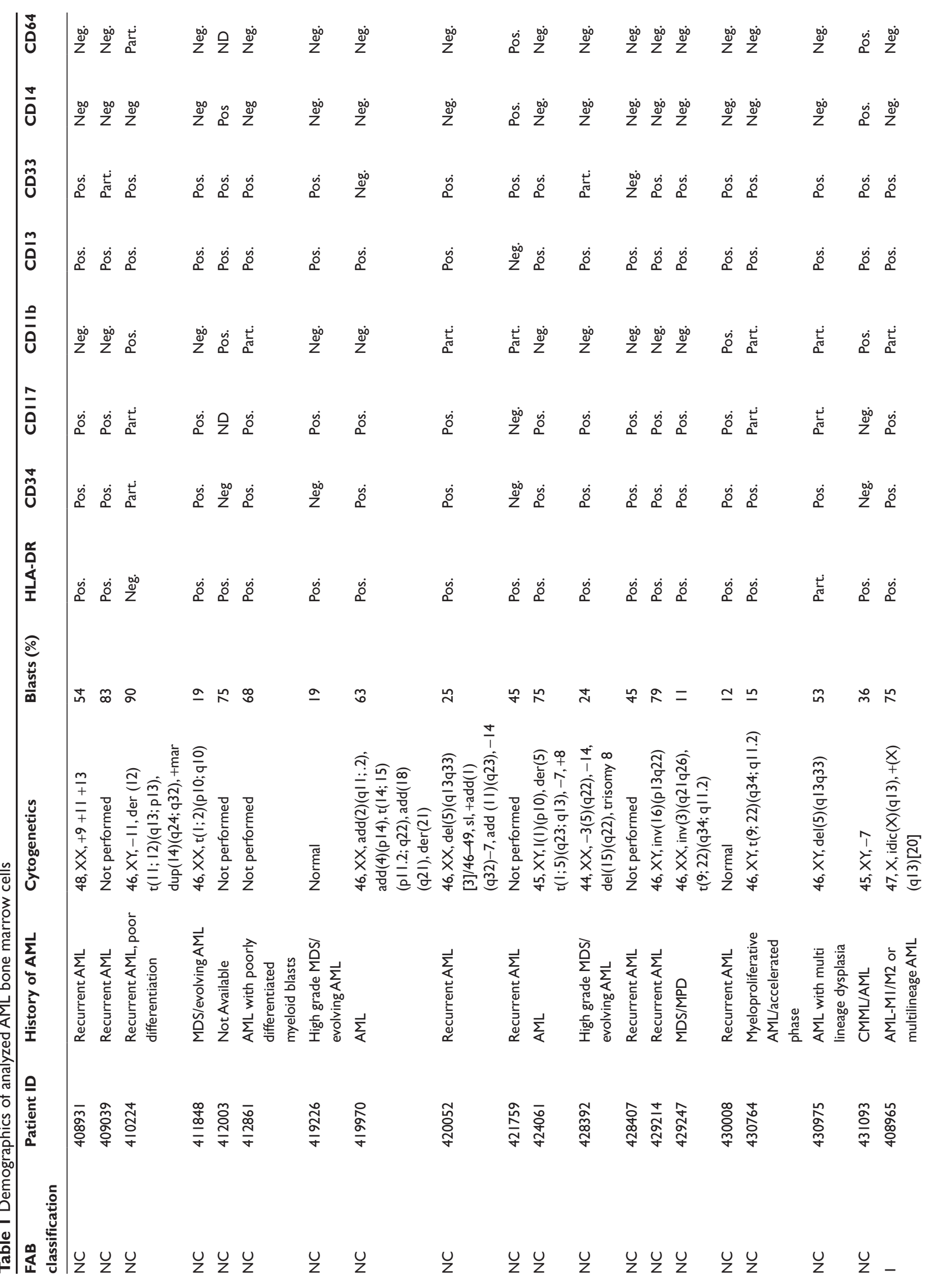




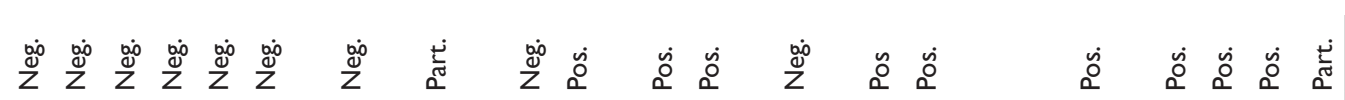

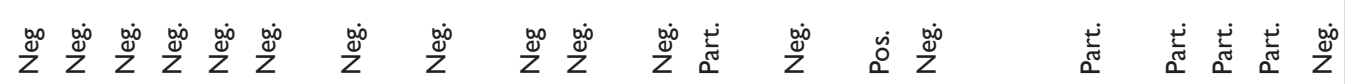

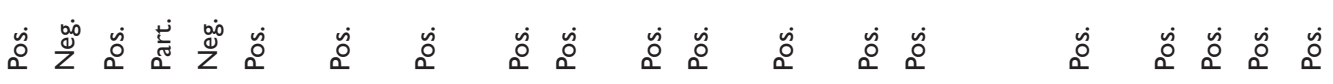

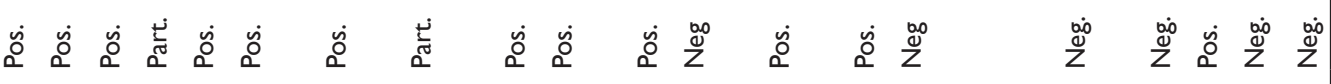

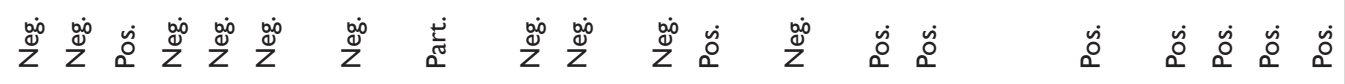
客咜

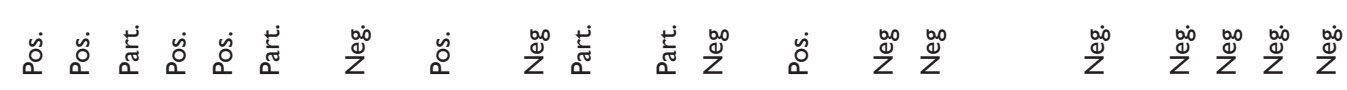

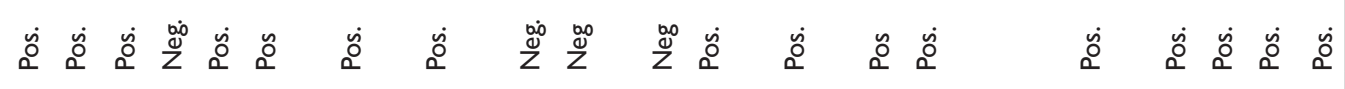
๓

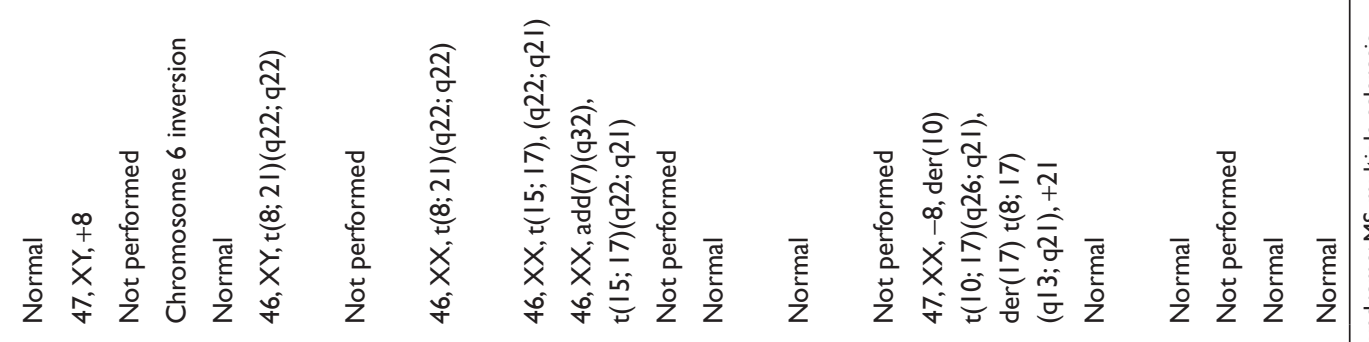

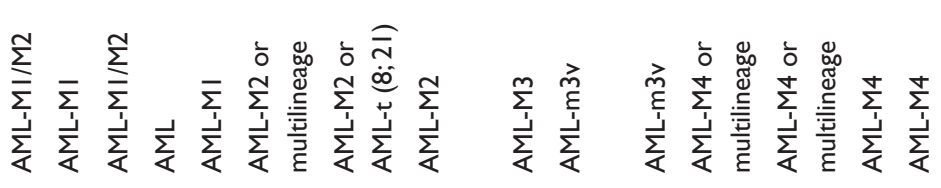

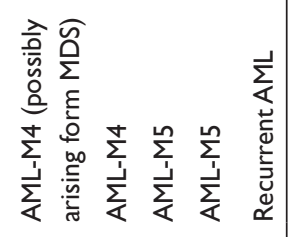

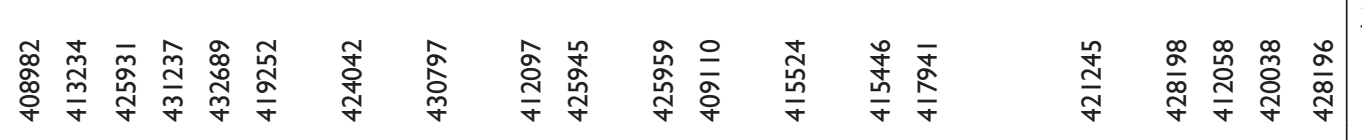



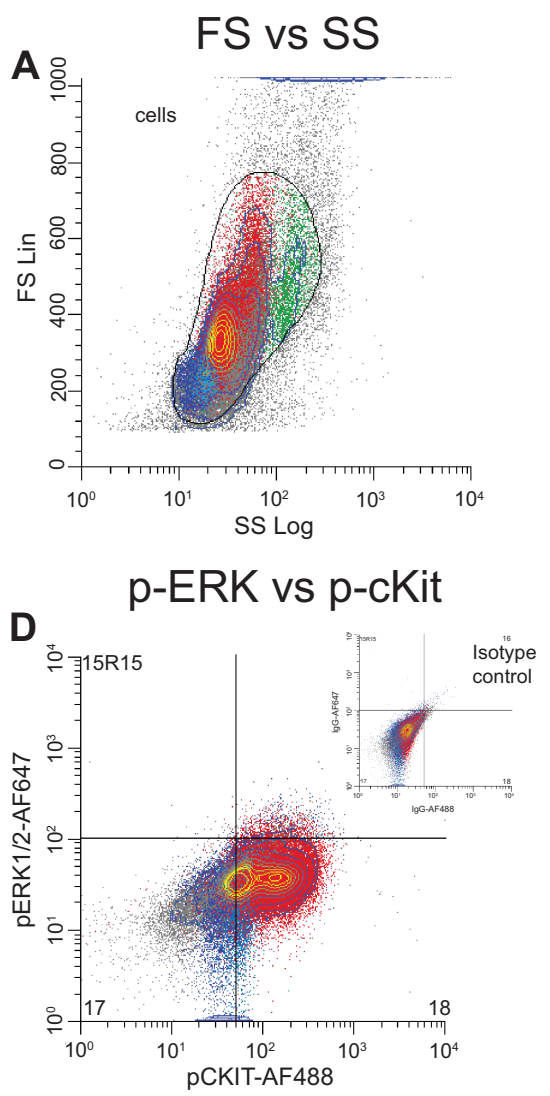
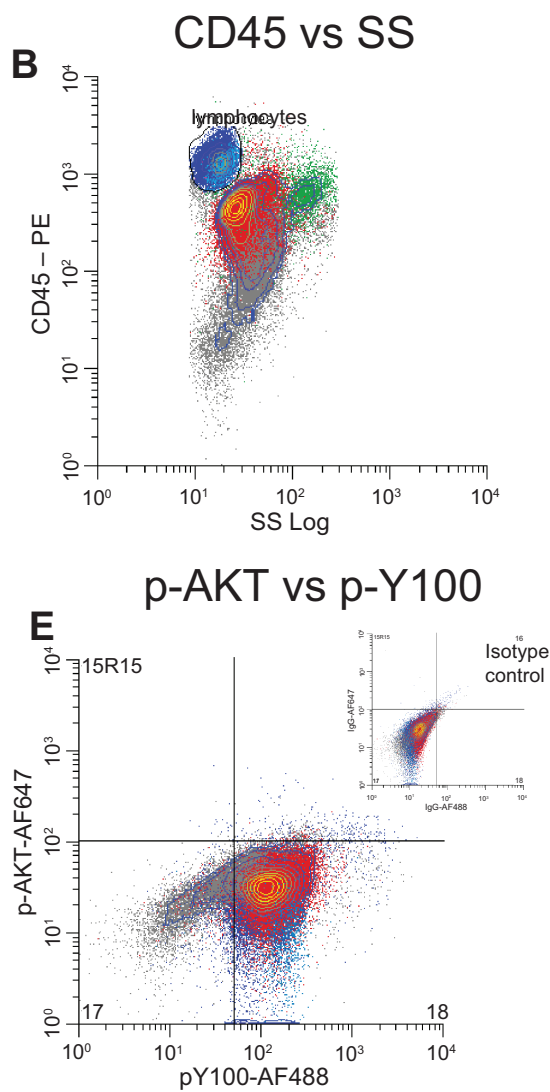
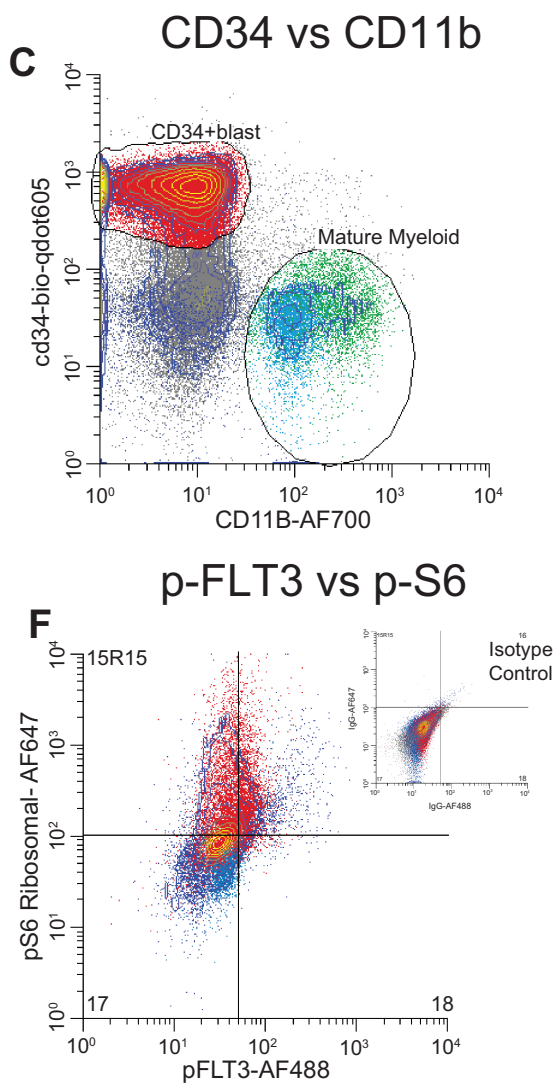

Figure I Detection of endogenous phospho-epitope levels. The combination of SSC/CD45/CD34/CDI Ib allows the delineation of the marrow elements (panels A-C). Cells were first assessed based on forward scatter (FS) versus side scatter (SS) (panel A), then grouped based on the CD45 and SS staining (panel B) and finally subdivided based on CD34 and CDI Ib staining (panel C). The lymphocytes (blue) lack CD34 and CDI Ib; the "blasts" (red) exhibit CD34 and lack CDI Ib whereas the maturing myeloid cells (green) exhibit CDI lb and lack CD34. After identifying the different subpopulation based on morphology (FS and SS) and surface marker staining (CD45/CDI lb/CD34), "blasts" were assessed for their expression of elevated levels of pc-KIT and pERK (panel D), pAKT and pYI00 (panel E) and pFLT3 and pS6. Isotype controls are inserted into each of the panels (cytograms D-F).

differences observed between phenotype 1 and 2 for p-FLT3 $(p=0.0034)$ and $p-Y 100(p=0.0041)$ (data not shown). There were also statistical differences observed in phospho-protein expression for p-FLT3 and p-Y100 among the AML FAB classification (Figure 3C). For these two IKs the M3 AML classification showed lower phospho-protein expression compared to the other subtypes (Figure 3, Panel C).

When the same AML cells were stimulated with SCF (Figure 4), the overall phospho-protein expression was modestly increased compared to un-stimulated cells (Table 3). In particular, pAKT, pS6 and pERK were significantly increased after stimulation with SCF compared to unstimulated cells (Table 3). Phospho-protein expression between phenotype 1 and 2 were similar to the overall pattern, but after stimulation there were statistical differences between phenotype for $\mathrm{p}$-ERK $(\mathrm{p}=0.0345)$, $\mathrm{p}$-FLT3 $(p=0.0047), p-Y 100(p=0.0095)$, and Survivin $(p=0.0186)$ (data not shown). Further, there were statistical differences observed in the phospho-protein expression for p-FLT3 among the AML FAB classification. For p-FLT3, the M3
AML classification was statistically lower than all other FAB classifications ( $<<0.05)$ (Figure 4C).

In contrast to phospho-protein expression of IKs, survivin belongs to a different group of intracellular signaling proteins and its overexpression has been associated with poor differentiation and prognosis in AML. ${ }^{19-21}$ Hence, the survivin expression served as an additional control in this study to differentiate between activation pattern of phospho-proteins and constitutive intracellular activation. Consistent with the previous studies, survivin expression was present in all AML cell and its expression did not increase after SCF stimulation (Figures 3 and 4).

Finally, we also compared the phospho-protein expression to the cells in the lymphocyte gate (Figure 5). We found that all phospho-protein levels are elevated and statistically different when compared to the cells in the lymphocyte gate. All IK signals increased by greater than 2-fold compared to lymphocytes with the greatest differences observed for pAKT (8-fold) and pERK (6-fold) (Figure 5A). This difference is remarkable because both phospho-proteins were low at the 

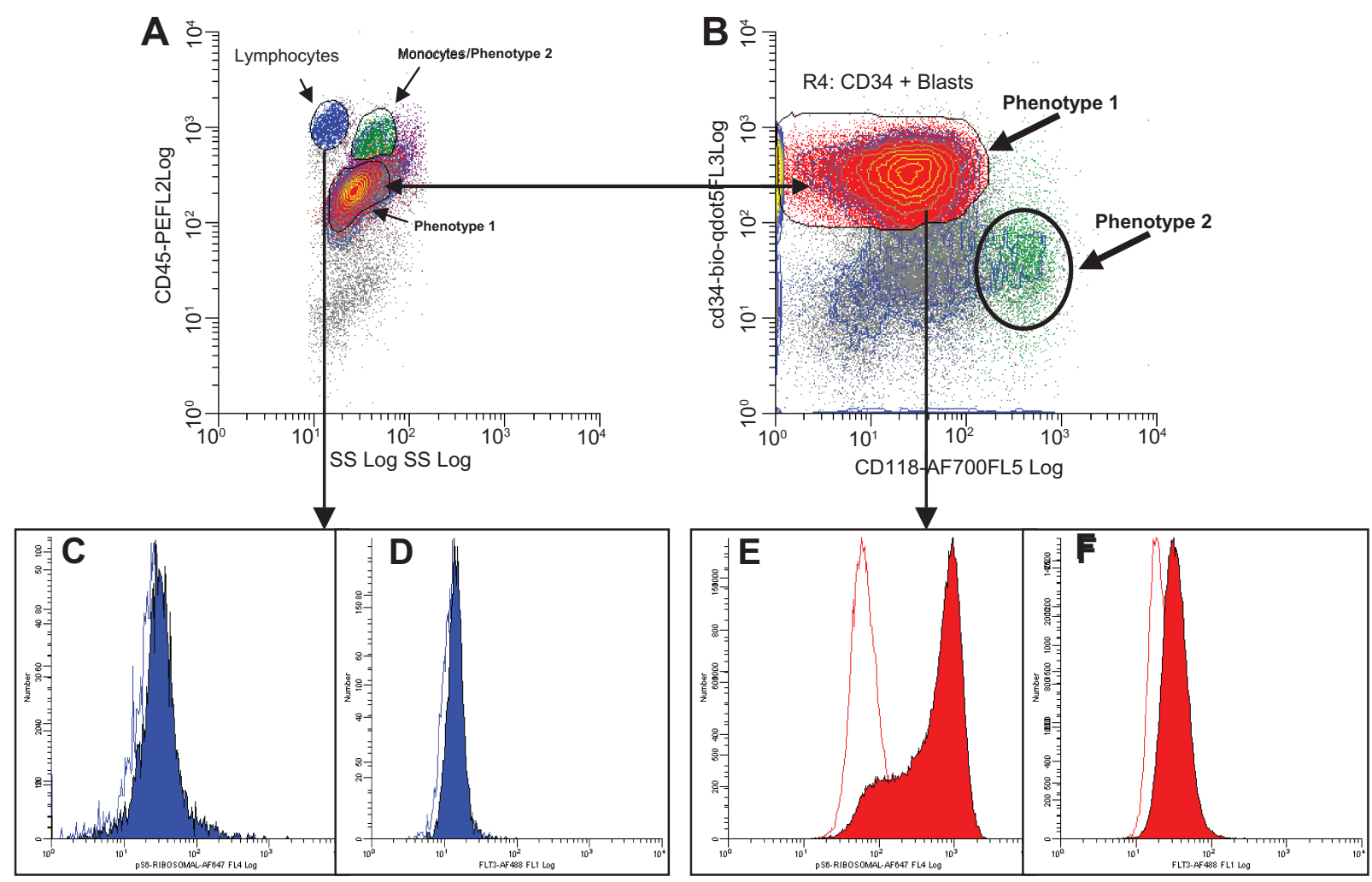

Figure 2 Phospho protein assessment in lymphocytes and AML (phenotype I) Cells. All cells above a light scatter trigger are displayed in panel A showing the cell population based on CD45 and Side Scatter (SS). Cells denoted by the color blue have dense CD45, low SS and lack both CD34 and CDI Ib, representing lymphocytes. In contrast, the cells denoted by the color red have moderate SS, low CD 45 and express CD34, representing the AML blast phenotype I. Green colored cells in panels A and B represent CD34 low AML blasts, which are denoted as phenotype 2. Panels C-F represent the phosphorylation status of pS6-ribosomal protein (panels C and E) and pFLT3 (panels D and F) for lymphocytes (blue color) and AML blasts of phenotype I (red color) in unstimulated (unfilled peaks) and after stimulated conditions with Stem cell factor (SCF) (I0 ng). Because of the marginal increase of phosphorylation in pS6 and pFLT3 after SCF stimulation, lymphocytes can serve as a negative control compared to AML blasts.

basal level for all the AML cells. Comparison to cells in the lymphocyte gate was even more useful to differentiate the phospho-protein expression pattern between phenotype 1 and 2 (Figure 5 B). Compared to the cells in the lymphocyte gate, phenotype 2 AML cells had higher phospho-protein levels than phenotype 1 with statistical differences observed for p-FLT3 (p = 0.0005) and p-Y100 (p=0.0005) (Figure 5B). For survivin, phenotype 1 was statistically lower than phenotype $2(p=0.0301)$. With regards to the FAB classification, M4 (7-fold, p = 0.0001) and M5 (12-fold, $p=0.0001)$

Table 2 Surface marker expression of AML cells allows definition of two categories (Phenotype I and 2)

\begin{tabular}{|c|c|c|}
\hline $\begin{array}{l}\text { Phenotype of } \\
\text { AML cells }\end{array}$ & $\begin{array}{l}\text { Characteristics } \\
\text { defined by } C D\end{array}$ & $\mathbf{N}$ (samples) \\
\hline Phenotype I & $\mathrm{CD} 4^{+} / \mathrm{CD} 45^{\mathrm{low}} / \mathrm{CD} / \mathrm{lb}^{-}$ & 40 \\
\hline Phenotype 2 & $\mathrm{CD} 34^{-} / \mathrm{CD} 45^{\mathrm{h} / \mathrm{mid} / \mathrm{CD}} / \mathrm{Ib}^{+}$ & 24 \\
\hline
\end{tabular}

Notes: For Phenotype I, 29 out of 40 samples were CD34+. For remaining II samples, CD34 was negative with concomitant CD45 staining being low. For Phenotype 2, 22 out of 24 samples were CD34-. All 24 samples from Phenotype 2 showed mid to high levels of CD45 staining. subtypes showed the greatest activation compared to the cells in the lymphocyte gate (Table 4). Thus, the comparison to cells in the lymphocyte gate appears to increase the resolution to differentiate phospho-protein expression in AML cells from other cell types present in the bone marrow.

\section{Discussion}

In AML, Western Blotting has been used to assess the inhibitory activity of IK inhibitors, such as sunitinib, ${ }^{8}$ and recently multiparametric analysis using flow cytometry has been applied to monitor phospho-protein changes after administration of IK inhibitors in patients with AML. ${ }^{16,22}$ The advantages of using flow cytometric analysis lie in its ability to provide quantitative assessment of cellular events and to determine pharmacodynamic effects in nearly realtime. In the present study, we evaluated the application of an assay to monitor phospho-protein changes in the setting of a clinical diagnostic pathology laboratory. The present assay is part of developing tools to monitor the pharmacodynamic changes induced by IK inhibitors in patients with AML. Thus, the validation comprised three important 


\section{A}

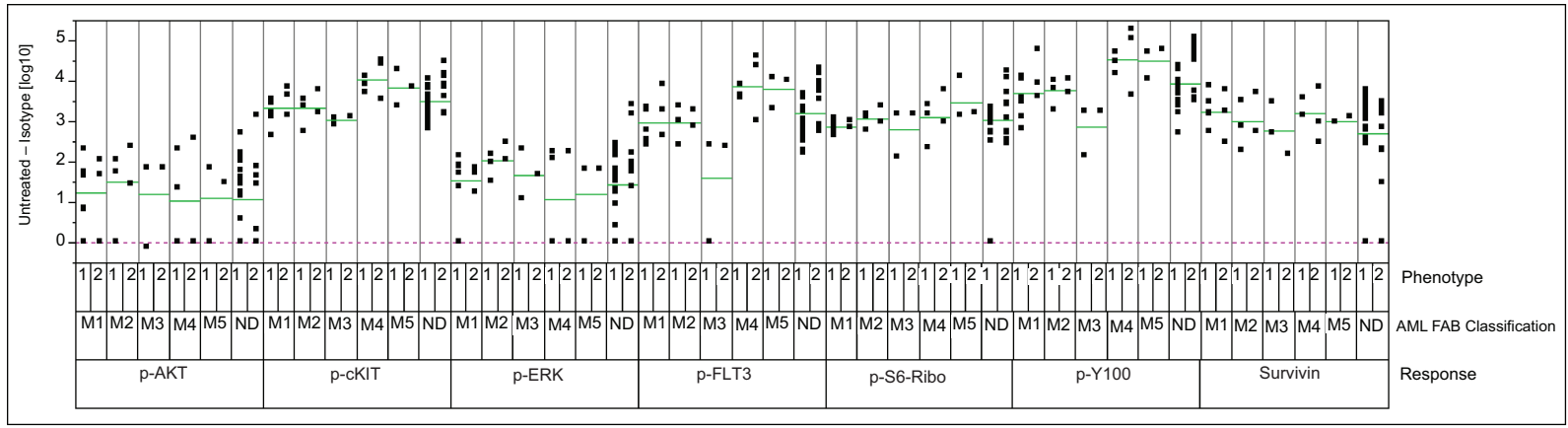

B

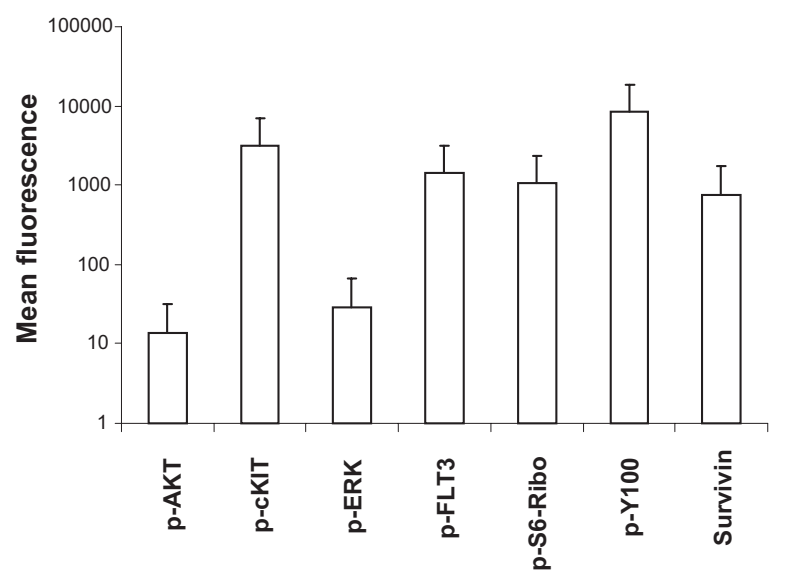

C

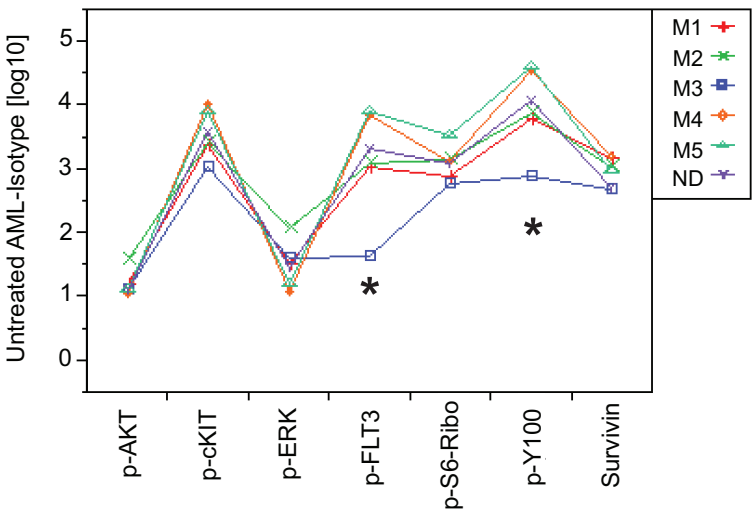

Figure 3 Constitutive phospho and survivin expression in AML cells $(n=40)$ without stimulation of stem cell factor (SCF). Panel A: Phospho-protein expression of individual samples represented as mean fluorescence value (log transformed and subtracted from isotype control) for pAKT, p-cKIT, pERK, pFLT3, pS6Ribo, pYI00, and survivin and classified by phenotype (I and 2) and FAB classification (x-axis). Panel B: Summary representation of phospho-protein and survivin expression. Expression of each phosphoprotein is represented as mean fluorescence after normalizing to isotype control. pAKT and pERK have lower expression than pc-KIT, pFLT3, pS6Ribo, PYI00 and survivin. Panel C: Phospho-protein expression classified by FAB AML classification. Expression of phospho-protein and survivin expression represented as mean fluorescence level (log transformed after subtracting values of isotype control) subtypes and plotted for each FAB AML subtype. Compared to all other subtypes, M3 AML cells have lower pFLT3 and PYI00 fluorescence.

development steps: first, the development of appropriate gating protocols to allow for simultaneous evaluation of various phospho-protein changes in patient-derived AML cells; second, a profile of phospho-protein expression across various types of AML cells (including those defined by the AML FAB classification); and thirdly, the differentiation of phospho-protein events from other cell population present in the bone marrow.

The present protocol allows the assessment of 6 different phospho-proteins in AML cells, including those that have been recently targeted by IK inhibitors (Figures 1 and 2). To our knowledge this is the first time that multiparametric assessment of both basal and stimulated levels of 6 different phospho-proteins has been reported. Setting up the appropriate gates involved the standard approach of defining the cell population based on size (FS vs SS), followed by the subsequent gating on surface marker expression defined by CD45, CD34 and CD11b. This generally is sufficient to gate on the most relevant AML population with myeloid characteristics, including "immature"- and "mature"-like phenotypes. A third population, which serves as a control, is the cell population in the lymphocyte gate, which is characterized by CD45 and $\mathrm{SS}^{\text {low }}$ expression. Our categories of phenotype 1 ("immature") and 2 ("mature") were made to reduce the complexity of the sample and to investigate whether AML cells with immature phenotype had a higher phospho-protein expression pattern than those with a more mature phenotype. Although, the "immature" AML cells had a higher phospho-protein expression than those with a more "mature-like" pattern, the numbers were too skewed towards the phenotype 1 to estimate a possible correlation of phenotype $1 / 2$ and a particular phospho-protein profile. The third gated population had the characteristics of lymphocytes. Although we have not formally demonstrated that this gate contains exclusively lymphocytes and given the recent observations of aberrant cell population in relapsed AML patients requiring careful 


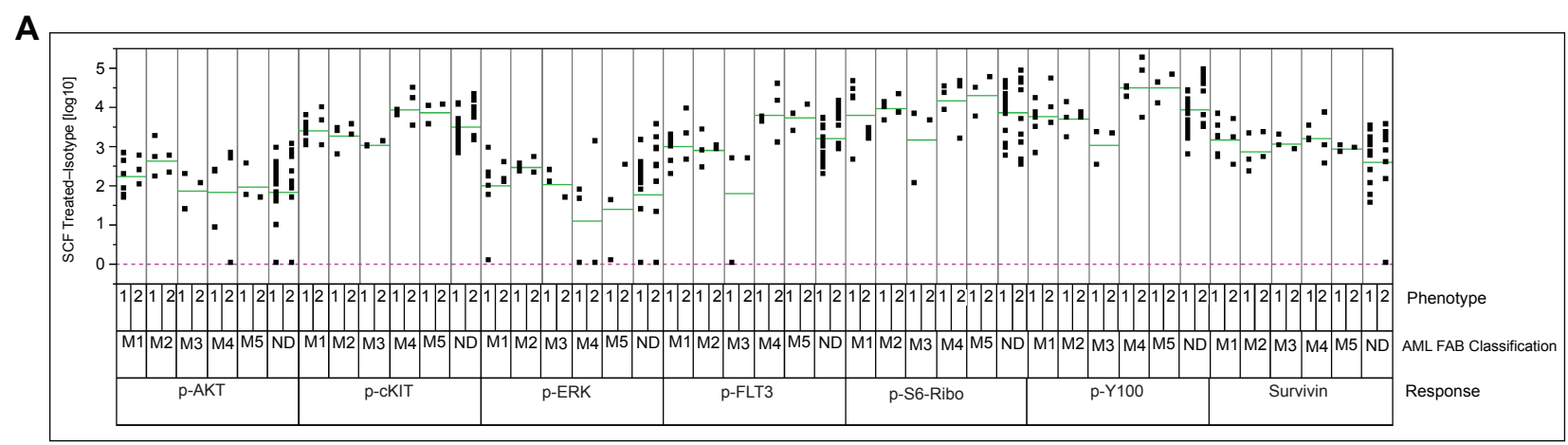

B

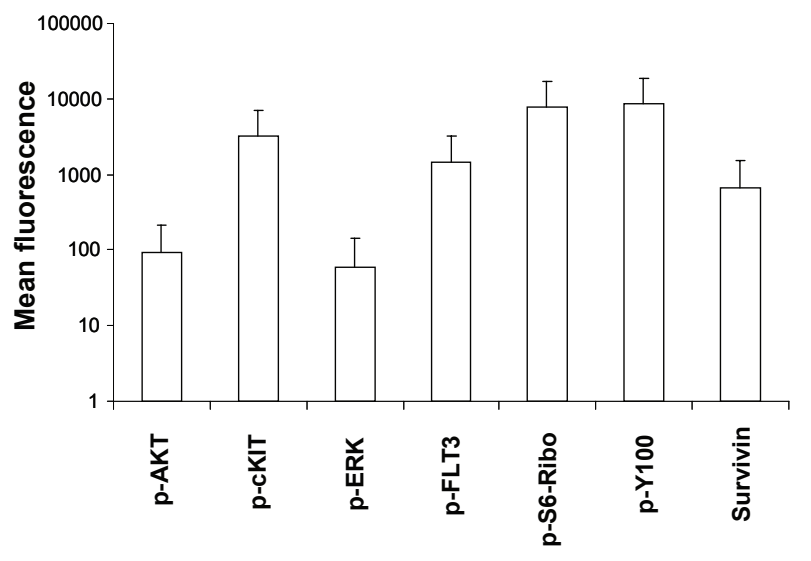

C

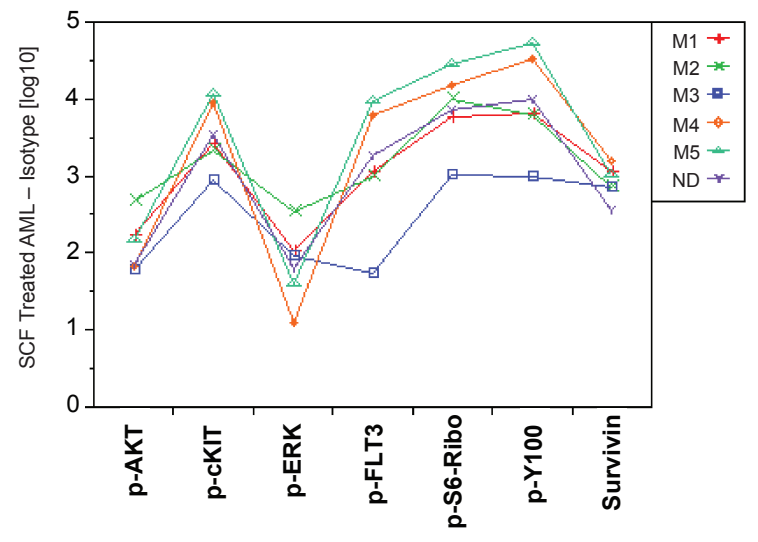

Figure 4 Constitutive phospho and survivin and expression in AML cells $(n=40)$ with stimulation of stem cell factor (SCF). Panel A: Phospho-protein expression of individual samples represented as mean fluorescence value (log transformed and subtracted from isotype control) for pAKT, p-cKIT, pERK, pFLT3, pS6Ribo, pYI00, and survivin and classified by phenotype (I and 2) and FAB classification (x-axis). Panel B: Summary representation of phospho-protein and survivin expression. Expression of each phosphoprotein is represented as mean fluorescence after normalizing to isotype control. pAKT and pERK have lower expression than pc-KIT, pFLT3, pS6Ribo, PYI00, and survivin. Panel C: Phospho-protein expression classified by FAB AML classification. Expression of phospho-protein and survivin expression represented as mean fluorescence level (log transformed after subtracting values of isotype control) subtypes and plotted for each FAB AML subtype. Compared to all other subtypes, M3 AML cells have lower pFLT3, pS6, and pYI00 fluorescence.

flow cytometry evaluation, ${ }^{23}$ prior studies have consistently demonstrated that this gate contains lymphocytes in patients with AML. ${ }^{24}$ This careful differentiation is important in order to attribute the phospho-protein profile to the AML cells. In the future, lymphocyte staining may be necessary if phospho

Table 3 Comparison of phospho-protein and survivin responses between unstimulated and SCF-stimulated AML cells

\begin{tabular}{lll}
\hline $\begin{array}{l}\text { Phospho-protein } \\
\text { and survivin } \\
\text { response }\end{array}$ & $\begin{array}{l}\text { Fold change } \\
\text { (SCF-stimulated/ } \\
\text { unstimulated) }\end{array}$ & $\begin{array}{l}\text { P-value (bold } \\
\text { are significant } \\
\text { changes) }\end{array}$ \\
\hline P-AKT & $\mathbf{8 . 2}$ & $<\mathbf{0 . 0 0 0 I}$ \\
P-ckit & 1.0 & 0.9733 \\
P-ERK & $\mathbf{2 . 2}$ & $\mathbf{0 . 0 2}$ \\
P-FLT3 & 1.0 & 0.9481 \\
P-S6 Ribo & 6.3 & $<\mathbf{0 . 0 0 0 I}$ \\
P-YI00 & 1.0 & 0.9129 \\
Survivin & 0.9 & 0.8019 \\
\hline
\end{tabular}

protein staining should also include the evaluation of changes in the lymphocyte population, such as lymphocytic leukemia. By using a semi-physiological stimulation for bone marrowderived cells with SCF, this selective stimulation of IKs in AML versus lymphocytes can be used to further support the identification of a unique phospho-protein expression in AML cells. The increased phospho-protein levels of pS6 and pAKT after stimulation with SCF is consistent with a differential activation of AML cells (Table 3). Lymphocytes do not typically have receptors associated with phosphorylation of S6 and AKT, which in turn explains that pS6 and pAKT were significantly increased after SCF stimulation (Table 3). Such increases have been also observed in patients with the ITD-FLT3 mutation (Shults, personal communication). By contrast, pERK activation has been observed in lymphocytic lineages or in activated lymphocytes. ${ }^{25,26}$ Despite the possibility that pERK activation could also be found in lymphocytes, in this study the 11-fold increase of pERK 
A

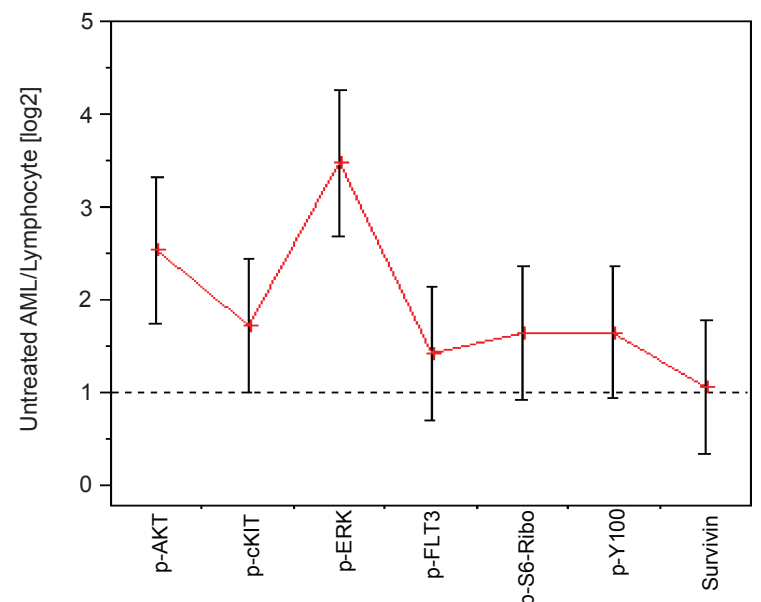

\begin{tabular}{|l|c|c|}
\hline \multicolumn{2}{|c|}{} & \multicolumn{2}{|c|}{ Response } & $\begin{array}{c}\text { Fold Change } \\
\text { (Untreated/ } \\
\text { Lymphocytes) }\end{array}$ & p value \\
\hline p-AKT & $\mathbf{5 , 8}$ & $<0.0001$ \\
\hline p-CKIT & 3,3 & $<0.0001$ \\
\hline p-ERK & $\mathbf{1 1 , 1}$ & $<0.0001$ \\
\hline p-FLT3 & 2,7 & 0,0001 \\
\hline p-S6-Ribo & 3,1 & $<0.0001$ \\
\hline p-Y100 & 3,2 & $<0.0001$ \\
\hline
\end{tabular}

B

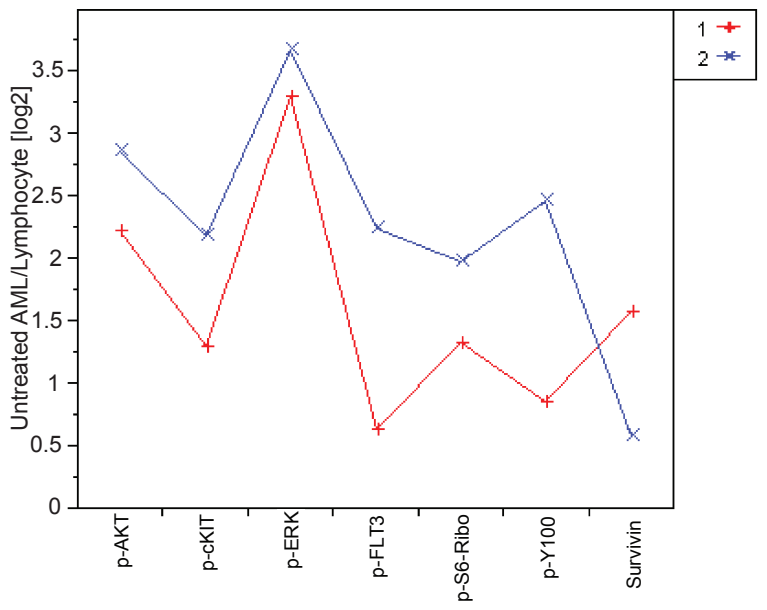

Figure 5 Panel A: Comparison of phospho-protein expression in AML blasts compared to lymphocyte population. Compared to lymphocytes, the expression of phospho-proteins is on average at least 2 -fold higher in AML blasts with PERK, PAKT, and pcKIT showing the highest difference (all responses are statistically different from 0 . Since the data are on the log2 scale and all the means are greater than I indicate that that all responses are greater than 2-fold higher than the lymphocytes). Panel B: Between the phenotype I and 2, phospho-protein expression ratio compared to lymphocytes is generally higher in AML phenotype 2 cells compared with phenotype I cells.

over lymphocytes and its increase after SCF stimulation, suggest that pERK was primarily activated in AML cells. Finally, vitality of AML blasts may influence the assessment of phospho protein expression in AML blasts. This may be of particular importance for shipping AML samples from the hematology practices to the test laboratory. Prior to conducting this study we performed a validation study of cell lines and 10 primary AML samples to investigated this

Table 4 Comparison of overall phospho-protein and survivin responses between unstimulated and lymphocytes

\begin{tabular}{lll}
\hline $\begin{array}{l}\text { AML subtype } \\
\text { (FAB classification) }\end{array}$ & $\begin{array}{l}\text { Fold change } \\
\text { (Untreated/ } \\
\text { Lymphocytes) }\end{array}$ & P-value \\
\hline M5 & 12.4 & 0.0001 \\
M4 & 7.1 & 0.0001 \\
ND & 3.5 & $<0.0001$ \\
M2 & 3.4 & 0.0086 \\
MI & 2.0 & 0.0411 \\
M3 & 1.5 & 0.4908
\end{tabular}

Abbreviation: ND, not defined. potential risk. We found that AML blasts had to be assessed within 24 to 48 hours after sampling in order to measure consistent and reproducible phospho protein levels (Shults, personal communication).

While recent guidelines for classifying AML recommend the use of the new World Health Organization (WHO) classification, ${ }^{27}$ the comparison of the phospho-protein expression to FAB AML subtypes may contribute to further re-fine outcome in AML patients (Table 1). Although the sample size in this study may be too limited to reach a final conclusion, in this set of data it appears that M4 and M5 subtypes exhibit a higher phospho-protein expression than patients with a M3 subtype. Whether this observation is consistent with previous reports suggesting that FLT3 expression is highest in M4 and M5 subtypes and thus is associated with a poor survival remains to be investigated. ${ }^{28}$

In conclusion, the present assay holds promise to detect pharmacodynamic changes in AML cells after treatment with a broadly active IK inhibitor and should be evaluated for its use in future clinical trials. However, the relevance of determining phospho protein levels as a prognostic marker 
was not the objective of this study and future prospective studies have to be conducted to determine its usefulness.

\section{Acknowledgments}

We would like to thank Drs Sada Pillay and Aimee Bence in supporting the development of this assay.

\section{Disclosures}

Lisa Green, Thomas Daly, Jason Manro and Michael Lahn are employed by Eli Lilly and Company. Keith Shults and Leanne Flye are employed by Esoterix, part of LabCorp.

\section{References}

1. Stone RM, O'Donnell MR, Sekeres MA. Acute myeloid leukemia. Hematology Am Soc Hematol Educ Program. 2004:98-117.

2. Correll PH, Paulson RF, Wei X. Molecular regulation of receptor tyrosine kinases in hematopoietic malignancies. Gene. 2006;374:26-38.

3. Kornblau SM, Tibes R, Qiu YH, et al. Functional proteomic profiling of AML predicts response and survival. Blood. 2009;113(1): 154-164.

4. DeAngelo DJ, Stone RM, Heaney ML, et al. Phase 1 clinical results with tandutinib (MLN518), a novel FLT3 antagonist, in patients with acute myelogenous leukemia or high-risk myelodysplastic syndrome: safety, pharmacokinetics, and pharmacodynamics. Blood. 2006;108(12): 3674-3681.

5. Fiedler W, Mesters R, Tinnefeld H, et al. A phase 2 clinical study of SU5416 in patients with refractory acute myeloid leukemia. Blood. 2003;102(8):2763-2767.

6. Fiedler W, Serve H, Dohner H, et al. A phase 1 study of SU11248 in the treatment of patients with refractory or resistant acute myeloid leukemia (AML) or not amenable to conventional therapy for the disease. Blood. 2005;105(3):986-993.

7. O'Farrell AM, Abrams TJ, Yuen HA, et al. SU11248 is a novel FLT3 tyrosine kinase inhibitor with potent activity in vitro and in vivo. Blood. 2003;101(9):3597-3605.

8. O'Farrell AM, Foran JM, Fiedler W, et al. An innovative phase I clinical study demonstrates inhibition of FLT3 phosphorylation by SU11248 in acute myeloid leukemia patients. Clin Cancer Res. 2003;9(15): 5465-5476.

9. O'Farrell AM, Yuen HA, Smolich B, et al. Effects of SU5416, a small molecule tyrosine kinase receptor inhibitor, on FLT3 expression and phosphorylation in patients with refractory acute myeloid leukemia. Leuk Res. 2004;28(7):679-689.

10. Stone RM, DeAngelo DJ, Klimek V, et al. Patients with acute myeloid leukemia and an activating mutation in FLT3 respond to a smallmolecule FLT3 tyrosine kinase inhibitor, PKC412. Blood. 2005; 105(1):54-60.

11. Kelly LM, Yu JC, Boulton CL, et al. CT53518, a novel selective FLT3 antagonist for the treatment of acute myelogenous leukemia (AML). Cancer Cell. 2002;1(5):421-432.
12. Tse KF, Novelli E, Civin CI, Bohmer FD, Small D. Inhibition of FLT3-mediated transformation by use of a tyrosine kinase inhibitor. Leukemia. 2001;15(7):1001-1010.

13. Pratz KW, Cortes J, Roboz GJ, et al. A pharmacodynamic study of the FLT3 inhibitor KW-2449 yields insight into the basis for clinical response. Blood. 2008. Nov 24. [Epub ahead of print].

14. Irish JM, Hovland R, Krutzik PO, et al. Single cell profiling of potentiated phospho-protein networks in cancer cells. Cell. 2004;118(2):217-228.

15. Perez OD, Nolan GP. Phospho-proteomic immune analysis by flow cytometry: from mechanism to translational medicine at the single-cell level. Immunol Rev. 2006;210:208-228.

16. Tong FK, Chow S, Hedley D. Pharmacodynamic monitoring of BAY 43-9006 (Sorafenib) in phase I clinical trials involving solid tumor and AML/MDS patients, using flow cytometry to monitor activation of the ERK pathway in peripheral blood cells. Cytometry B Clin Cytom. 2006;70(3):107-114.

17. Krutzik PO, Nolan GP. Intracellular phospho-protein staining techniques for flow cytometry: monitoring single cell signaling events. Cytometry A. 2003;55(2):61-70.

18. Wang L, Gaigalas A, Abbasi F, Marti G, Vogt R, Schwartz A. Quantitating fluorescence intensity from fluorophores: practical use of MESF values. J Res Natl Inst Stand Technol. 2002;107:339-353.

19. Adida C, Recher C, Raffoux E, et al. Expression and prognostic significance of survivin in de novo acute myeloid leukaemia. Br J Haematol. 2000;111(1):196-203.

20. Tamm I, Richter S, Oltersdorf D, et al. High expression levels of $\mathrm{x}$-linked inhibitor of apoptosis protein and survivin correlate with poor overall survival in childhood de novo acute myeloid leukemia. Clin Cancer Res. 2004;10(11):3737-3744.

21. Oto OA, Paydas S, Tanriverdi K, Seydaoglu G, Yavuz S, Disel U. Survivin and EPR-1 expression in acute leukemias: prognostic significance and review of the literature. Leuk Res. 2007;31(11):1495-1501.

22. Chow S, Minden MD, Hedley DW. Constitutive phosphorylation of the S6 ribosomal protein via mTOR and ERK signaling in the peripheral blasts of acute leukemia patients. Exp Hematol. 2006;34(9):1182-1191.

23. Craig FE, Foon KA. Flow cytometric immunophenotyping for hematologic neoplasms. Blood. 2008;111(8):3941-3967.

24. Borowitz MJ, Guenther KL, Shults KE, Stelzer GT. Immunophenotyping of acute leukemia by flow cytometric analysis. Use of CD45 and right-angle light scatter to gate on leukemic blasts in three-color analysis. Am J Clin Pathol. 1993;100(5):534-540.

25. Adams CL, Grierson AM, Mowat AM, Harnett MM, Garside P. Differences in the kinetics, amplitude, and localization of ERK activation in anergy and priming revealed at the level of individual primary $\mathrm{T}$ cells by laser scanning cytometry. J Immunol. 2004;173(3):1579-1586.

26. Milne CD, Corfe SA, Paige CJ. Heparan sulfate and heparin enhance ERK phosphorylation and mediate preBCR-dependent events during B lymphopoiesis. J Immunol. 2008;180(5):2839-2847.

27. Brunning RD, Matutes E, NL H. Acute myeloid leukaemia: Pathology and Genetics of Tumours of Hematopoietic and Lymphoid Tissues. Lyon, France: IARC Press; 2001.

28. Thiede C, Steudel C, Mohr B, et al. Analysis of FLT3-activating mutations in 979 patients with acute myelogenous leukemia: association with FAB subtypes and identification of subgroups with poor prognosis. Blood. 2002;99(12):4326-4335.
Cancer Management and Research

\section{Publish your work in this journal}

Cancer Management and Research is an international, peer-reviewed open access journal focusing on cancer research and the optimal use of preventative and integrated treatment interventions to achieve improved outcomes, enhanced survival and quality of life for the cancer patient. The journal welcomes original research, clinical \& epidemiological

\section{Dovepress}

studies, reviews \& evaluations, guidelines, expert opinion \& commentary, case reports \& extended reports. The manuscript management system is completely online and includes a very quick and fair peerreview system, which is all easy to use. Visit http://www.dovepress.com/ testimonials.php to read real quotes from published authors. 